\title{
Fabrication of Zinc-Iron-Aluminum Layered Double Hydroxides with Controlled Micromorphology in a Tailored Aqueous - Organic Synthesis Medium
}

\author{
DORINA STANCIULESCU ${ }^{1}$, GABRIELA CARJA ${ }^{1}$, DOINA LUTIC ${ }^{2 *}$ \\ "Gheorghe Asachi" Technical University of Iasi, Faculty of Chemical Engineering and Environmental Protection, \\ Department of Chemical Engineering, 73 Prof. dr. docent Dimitrie Mangeron Str., 700050, Iasi, Romania \\ 2"Alexandru Ioan Cuza" University of Iasi, Faculty of Chemistry 11 Carol I Blvd., 700506, Iasi, Romania
}

\begin{abstract}
Micromorphology characteristics of layered double hydroxides are important to establish their performances to act as adsorbents and catalysts/photocatalysts. Herein, we report the fabrication

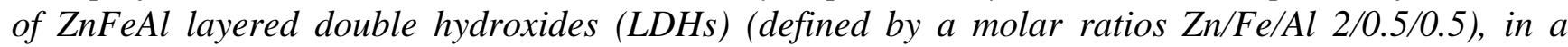
tailored aqueous-organic synthesis medium, by using the coprecipitation method. The nature of the organic solvent (e.g.: ethanol, ethyl acetate, glycerol and toluene) was used as a controlled variable for obtaining specific morphology characteristics of ZnFeAl. XRD, FTIR, UV-Vis-DR spectroscopy and SEM microscopy have been used to characterize the structural, optical and textural features of ZnFeAl. Results show that the controlled aqueous-organic synthesis medium established important changes in the structural features and the micromorphology characteristics (e.g.: particle sizes and their interconnection patterns) of ZnFeAl, as indicated by the results of XRD and SEM analyses. The FTIR spectra revealed that most of the interlayer anions are carbonate, even though the initial salts precursors contain nitrates or sulfates anions. Furthermore, the UV-Vis-DR results underline that the band gaps of the LDH derived oxides changed in a significant manner, as a function of the nature of the organic solvent. Such that, ZnFeAl prepared in the presence of ethanol and ethyl acetate show band gap values lower than $3 \mathrm{eV}$ and thus could be better activated under visible light. These results should be inspiring for fabricating layered double hydroxides with a controlled micromorphology for specific applications for environmental cleanup.
\end{abstract}

Keywords: layered double hydroxides, controlled micromorphology, organic solvents

\section{Introduction}

The environment pollution is nowadays a constant concern for scientists, political and administration leaders and even for common people. In this respect, the water resources are constantly aggressed by spilling pesticides, pharmaceuticals (drugs, personal care products, disinfectants, food and medicines preservatives), detergents and other surfactants, halogen-containing compounds and plasticizers from plastic products, waste from oil processing and many other chemicals in wastewaters or by the leakage of pollutants from the landfill deposits of solid waste [1,2]. Since the water pollution cannot be entirely avoided, the preparation of potable water needs special attention, especially when the available source are big rivers collecting in their flowing course wastewater outpoured upstream. In this context, there is a high interest to develop performant materials for the adsorption and/or (photo)catalytic transformation of low concentrations of harmful and stable compounds from wastewaters [3]. Layered double hydroxides (LDHs) are a class of anionic clays that are emerging as suitable candidate for cleaning the water due to their performant adsorbent and catalytic or photocatalytic properties [4-10]. Their cost-effective synthesis methods, using "green" precursors, are another reason for the interest of the scientific community as well as that of companies specialized in adsorbents and catalysts manufacturing.

\footnotetext{
*email: doilub@uaic.ro
} 
The performances of a material used as adsorbent or (photo)catalyst are related to the micromorphology properies [11]. The performance increases when the surface area and the textural features afford an accessible adsorption of different species from solutions [12].

This work presents how the micromorphology features of ZnFeAl LDHs, obtained by the coprecipitation method, can be controlled by tailoring a specific aqueous-organic synthesis medium. Thus, we show for the first time the contribution of the organic solvent for establishing a specific micromorphology features for $\mathrm{ZnFeAl} \mathrm{LDH}$, defined by a molar ratio of $\mathrm{Zn} / \mathrm{Fe} / \mathrm{Al}=2 / 0.5 / 0.5$. Ethanol, ethyl acetate, glycerol and toluene are used as specific organic solvents and the molar ratio of water/organic solvents is $3 / 1$. The effect of the organic solvents is further highlighted by the changes of the XRD patterns, micromorphology characteristics and further in the decrease of the band gap of the semiconductive oxides. By controlling the characteristics and especially the micromorphology of the ternary metals LDH open the opportunities to fabricate more performant catalysts that are active in a wide-range domain. Further, the low toxicity level of these materials is offering an extra-advantage in the perspective of their use for water decontamination.

\section{Materials and methods}

\subsection{Synthesis}

The $\mathrm{ZnFeAl} \mathrm{LDH}$ with a molar ratio of $\mathrm{Zn} / \mathrm{Al} / \mathrm{Fe}$ of $2 / 0.5 / 0.5$ is synthesized by the coprecipitation method. $\mathrm{Zn}$ and $\mathrm{Al}$ sources are based on nitrates salt precursors while Fe was used as nitrate and sulfate (all from Sigma-Aldrich) salts. The metal salt precursors were dissolves in distilled water, then mixed to reach a total concentration of $1 \mathrm{M} ; 0.1$ moles of $\mathrm{Zn}$ nitrate and 0.025 moles of each salt of $\mathrm{Fe}$ and $\mathrm{Al}$ were used. This solution was poured in a beaker, under continuous magnetic stirring at 300 rpm, into a $\mathrm{Na}_{2} \mathrm{CO}_{3}$ solution, then $\mathrm{NaOH}$ solution (2M) was added in small drops to keep the $\mathrm{pH}$ of the reaction medium at a constant value of $8.5 \pm 0.2$ (monitored by a Hanna HI99301 instrument), by manipulating the ratio between the flows of metallic salts and the $\mathrm{NaOH}$ solution. The flowrate of the mixture of metallic salts was $5 \mathrm{~mL} \cdot \mathrm{min}^{-1}$. In the meantime, several organic solvents were poured together with the salts solution, in proper flow rates, to reach a 3/1 molar ratio water/solvent. The solvents used were ethanol (sample denoted as EtOH), ethyl acetate (sample denoted EtOAc), glycerol (sample denoted as Gly) and toluene (the sample is denoted as Tol). The Gly and Tol samples were prepared using Fe sulfate, while for $\mathrm{EtOH}$ and EtOAc, nitrate was used. The resulted slurry was aged about $12 \mathrm{~h}$ at room temperature $\left(23^{\circ} \mathrm{C}\right)$, then the solid was separated by filtration and washed several times with warm water. The solids resulted after this procedure were filtered, dried at room temperature overnight, then under vacuum at $80^{\circ} \mathrm{C}$ for 4 hours.

\subsection{Characterization techniques}

The phase identification from the samples was performed by powder X-ray diffraction (XRD) on a Shimadzu XRD 6100 diffractometer using the CuK $\alpha$ radiation $(\lambda=0.1541 \mathrm{~nm})$, operated over a $2 \theta$ range $4-70^{\circ}$. The structure constants were calculated by the relations: $a=2 \mathrm{~d}_{[110]}$ and $c=3 \mathrm{~d}_{\text {[003] }}$ (the indexes indicate the orientation of the two characteristic planes from the XRD diffraction pattern), adequate for the rhombohedral symmetry. The diffuse reflectance (DR) spectra were recorded on a Jasco V550 spectrophotometer with integration sphere in the wavelength range $200-900 \mathrm{~nm}$, using magnesium oxide as dilution solid. The Fourier transform infrared (FTIR) spectra were collected on a Spectrum 100 spectrophotometer (Perkin Elmer) in the range $450-4000 \mathrm{~cm}^{-1}$, on $\mathrm{KBr}$ pellets. The SEM images were obtained on a Mira Tescan Type Mira II LMU device.

\section{Results and discussions}

\subsection{Structural characterization}

The XRD patterns of the samples, displayed in Figure 1, confirm that the layered structure of the solids was achieved during the synthesis for all reaction systems containing organic solvents (ethanol, ethyl acetate, glycerol and toluene). 


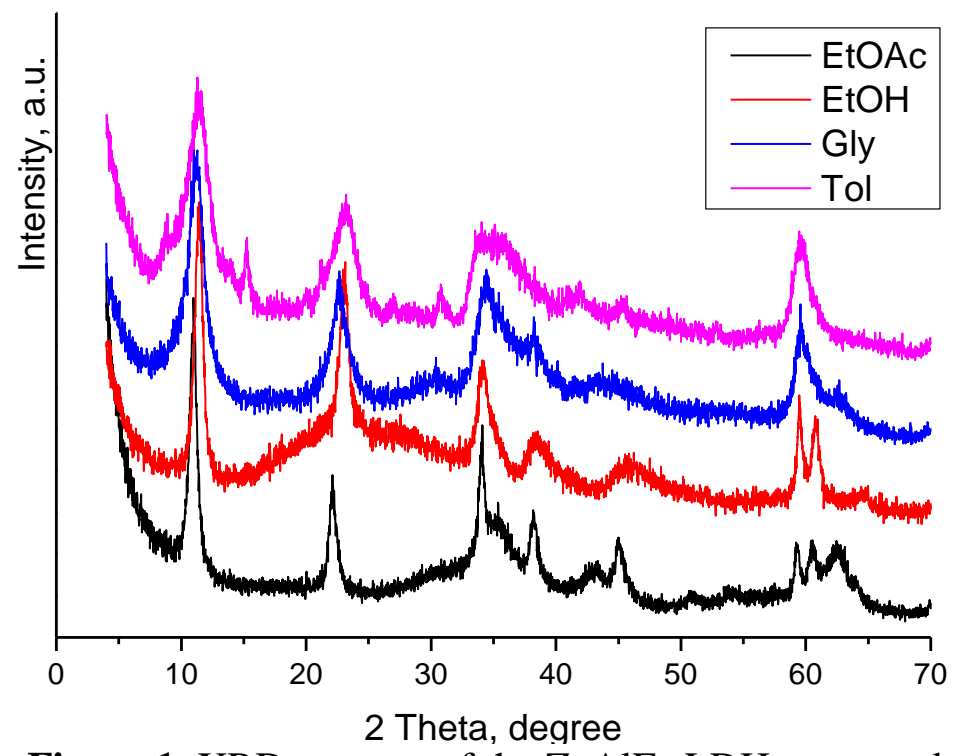

Figure 1. XRD patterns of the ZnAlFe LDHs prepared in water-organic solvents systems

The planes indexation (Table 1) indicate that the double layered structure is confirmed, since the main planes characteristic to LDHs were found for all samples, but the structures are largely distorted in comparison with samples having similar network composition, prepared in aqueous medium only [13].

Table 1. Structural data delivered by XRD

\begin{tabular}{cccccccccccc}
\hline $\begin{array}{c}\text { Sample/ } \\
\text { Planes }\end{array}$ & {$[003]$} & {$[006]$} & {$[101]$} & {$[009]$} & {$[015]$} & {$[107]$} & {$[110]$} & {$[113]$} & $a, \mathrm{~nm}$ & $c, \mathrm{~nm}$ & $\begin{array}{c}\text { IS*, } \\
\mathrm{nm}\end{array}$ \\
\hline EtOAc & 10.98 & 22.10 & 34.06 & 34.12 & 38.24 & 45.06 & 59.40 & 62.58 & 3.109 & 24.15 \\
EtOH & 11.44 & 22.98 & 34.16 & 34.16 & 38.48 & 45.46 & 59.56 & 60.80 & 3.044 & 23.19 & 2.95 \\
Gly & 11.22 & 22.72 & - & 34.52 & 38.20 & 43.78 & 59.62 & - & 3.099 & 23.64 & 3.10 \\
Tol & 11.40 & 22.40 & - & 34.28 & 36.34 & 42.10 & 59.56 & - & 3.102 & 23.27 & 2.97 \\
\hline \multicolumn{8}{c}{ *IS, interlammelar space, IS $=$ d[003]-4.78 (brucite layer thickness) } & &
\end{tabular}

The structural details presented in Figure 1 and Table 1 indicate that the use of organic solvents generates powder diffraction patterns with rough baseline and low intensity, wide peaks, characteristic to the presence of large ratios of amorphous solid. The positions of the maxima prove that the LDH structure was formed in all samples, as shown by the presence of [003], [006] and [110] planes. However, the peaks corresponding to planes [101] and [113] are missing totally and the intensity of the peak due to [107] plane is less intense and tailed for Gly and Tol samples. In the meantime, the width of the [003], [006] and [110] planes is much larger and their height is lower. The effects of glycerol and toluene on the LDH structure are stronger than these of ethanol and ethyl alcohol. Indeed, the main peaks of LDH are better defined for samples EtOH and EtOAc, though the baseline is still not horizontal and straight. In the pattern of EtOH sample, an extra peak situated around $63^{\circ}$ could be assigned to [103] plane of $\mathrm{ZnO}$; this is in line with the evident peaks overlap occurring in the angle range of $32-37^{\circ}$, where the presence of the [100], [002] and [101] define a triplet of peaks [14]. The values of the " $a$ " and " $c$ " parameters, calculated for the rhombohedral symmetry, indicate that the thickness of the brucite-type layer varies very little with the solvent. The $c$ parameter shows similar values, showing that the samples are quite similar in this respect. This reveals that the structure distortion of the octahedral packed units associated to the presence of $\mathrm{Zn}, \mathrm{Fe}$ and $\mathrm{Al}$ is quite minor; these values are very close the ones previously calculated for both $\mathrm{Mg}$ - $\mathrm{Al}$ and $\mathrm{Zn}-\mathrm{Fe} \mathrm{LDHs}$. Indeed, the $a$ and $c$ values for Mg-Al LDHs were 3.099 and 23.473, while for Zn-Fe LDHs, $a$ and $c$ were found 
to be $3.063 \mathrm{~nm}$ and 23.724, respectively [15]. The compactness of the interlayer anionic space is better highlighted by the value of the interlayer space (IS), calculated by subtracting the thickness of the brucite-like layer $(4.78 \mathrm{~nm})$ [16]. The IS values are slightly higher for EtOAc and Gly, indicating their better ability to expand the interlayer space.

The FT-IR spectra of the samples are represented in Figure 2. The large band at around $3450 \mathrm{~cm}^{-1}$ is due to stretching vibration of $\mathrm{OH}$ groups from the brucite-like layers and to the interlamellar water molecules. The very weak band at $1380 \mathrm{~cm}^{-1}$ is due to the stretching vibration of the $\mathrm{NO}_{3}{ }^{-}$groups from the $\mathrm{LDH}$ interlayer space, the intense one at $1353 \mathrm{~cm}^{-1}$ is given by the $\mathrm{CO}_{3}{ }^{2-}$ groups, while at $1100 \mathrm{~cm}^{-}$ ${ }^{1}$ the signal is due to the $\mathrm{SO}_{4}{ }^{2-}$ groups, acting as compensation anions in the interlayer. The high affinity of the carbonate anion for accommodating in the interlayer space of LDHs as compensating anion is confirmed by the high ratio between the intensity of the carbonate ions versus the intensity of sulfate ions. Also, the nitrate ions present in EtOH and EtOAc samples are in very low number compared with carbonate ions. The treatment in non-aqueous solvents seems to favor the diffusion of carbon dioxide from air, in the ionized form of carbonate ion, to the interlayer space and progressively replace almost totally the nitrate ions which were present in the initial precipitation medium from the precursor salts.

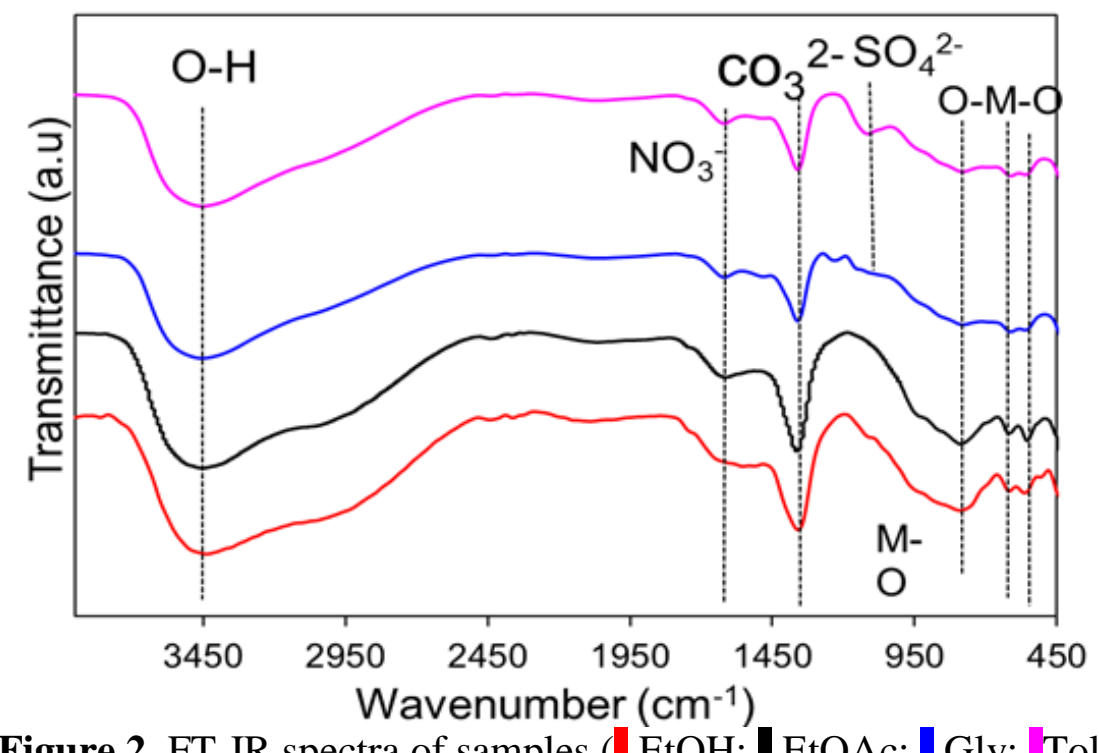

Figure 2. FT-IR spectra of samples (- EtOH; |EtOAc; | Gly; -Tol)

The association of zinc and iron as oxides in a LDH-type material, two species of semiconductive oxides, are an extra-premise for obtaining semiconductive materials that could be used as active photocatalysts activated by solar light. Indeed, the diffusion reflectance spectra traced in the 200-900 $\mathrm{nm}$ range, allow determining the band gap energy for these solids, by the extrapolation of the linear portion of the spectrum up to crossing the abscissa. The mathematical processing of the data as Tauc plots (Figure 3) improves the accuracy of the determination and delivers directly the band gap (BG) energies in $\mathrm{eV}$ [17]. The estimation of the band gap energy from an optical absorption spectrum from the Tauc relationship consists in the graphical representation of the function $(\alpha h v) \propto(h v-B G)^{n}$, where $\mathrm{h}$ is the Planck's constant, $v$ is the frequency of light and BG is the band gap energy and $\mathrm{n}$ is a value showing the electronic nature of the band gap; its values are 3,2,3/2, and $1 / 2$ corresponding to indirect forbidden (IF), indirect allowed (IA), direct forbidden (DF), and direct allowed (DA) transitions of the electrons. In the case of semiconductive materials, the BG value is determined by extrapolating the linear portion in the representation of $(\alpha h v)^{1 / n}(n=1 / 2)$ versus $h v$ plots (Tauc plot) up to meet the abscisa. The Tauc plots for our series of samples is given in Figure 3. 


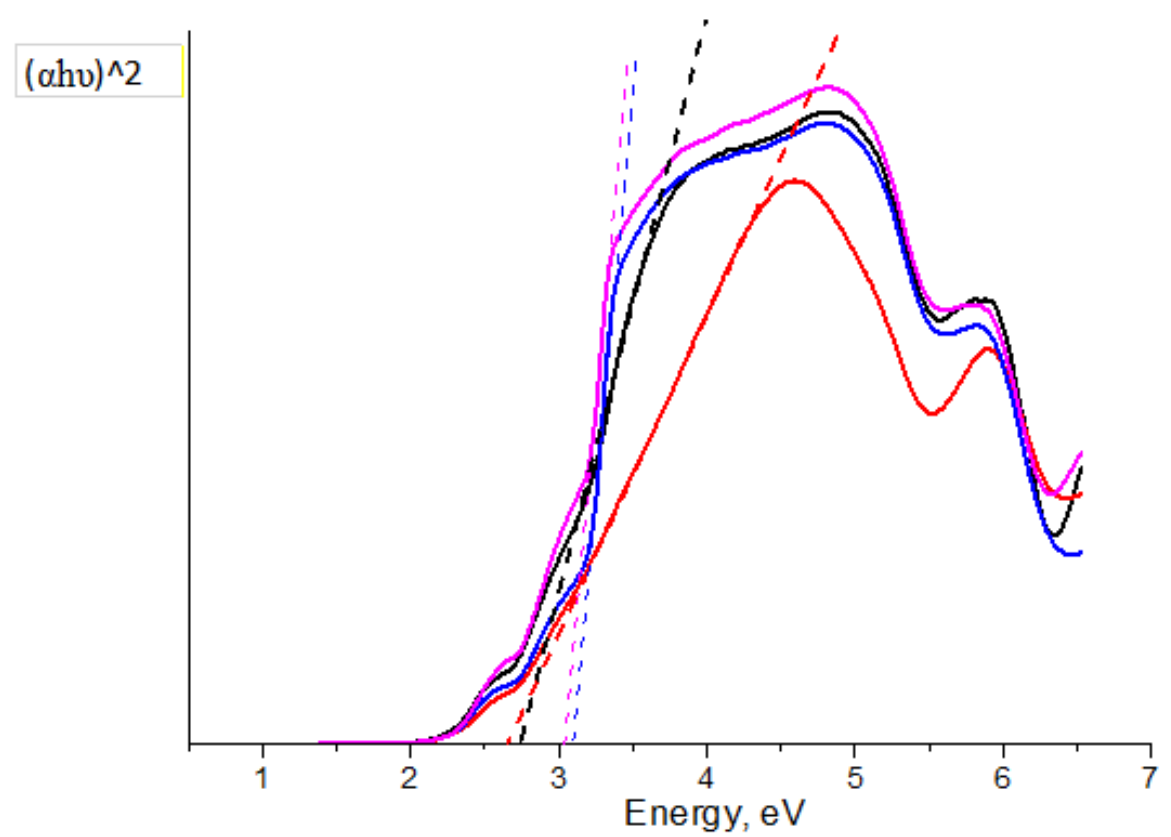

Figure 3. Tauc plots on the basis of the UV- Vis DR spectra of the samples (-EtOH; EtOAc; Gly; -Tol)

The band gap value (BG), in $\mathrm{eV}$, is connected to the light wavelength (in $\mathrm{nm}$ ) able to activate the electron promotion from the valence band to the conduction band by the relation:

$$
\lambda=1240 / \mathrm{BG}
$$

The BG values highlighted on Fig. 3, of $2.65 \mathrm{eV}$ (EtOH), $2.75 \mathrm{eV}$ (EtOAc), $3.15 \mathrm{eV}$ (Gly) and 3 $\mathrm{eV}$ (Tol) correspond to light wavelength of: $468 \mathrm{~nm}$ (EtOH), $450 \mathrm{~nm}$ (EtOAc), $393 \mathrm{~nm}$ (Gly) and 413 $\mathrm{nm}$ (Tol), respectively. The values higher than $400 \mathrm{~nm}$ correspond to the visible light range. So, the EtOH, EtOAc and Tol samples can be activated by visible light, meaning that the promotion of an electron from the valence band to the conduction band can be promoted under solar irradiation. This is a premise for the decomposition of stable organic pollutants from wastewaters, by using the solar energy - readily available and green source - for the advanced oxidation (AOPs) water treatment, in the presence of such solids used as photocatalysts.

Together with the band gap value which can be activate by natural light, another requirement for the solids used in heterogeneous (photo)catalysis is their morphology and texture. A successful solid for a catalytic application should be able to receive the activating light on as many as possible exposed particles, but also be a good adsorbent for the interaction with the target pollutant. The organic molecule is oxidized during a surface reaction, from adsorbed state. The success key remains however the generation of numerous $\mathrm{HO}^{\bullet}$ radicals, resulting from the interaction between water adsorbed on the solid and a hole formed on the solid after the electron promotion from the valence to the conduction band. Therefore, the solids with a scattered appearance of the particles are more suited for these kinds of processes.

The investigation of the solid morphology was performed by SEM microscopy. Some representative images are shown in Figure 4. 

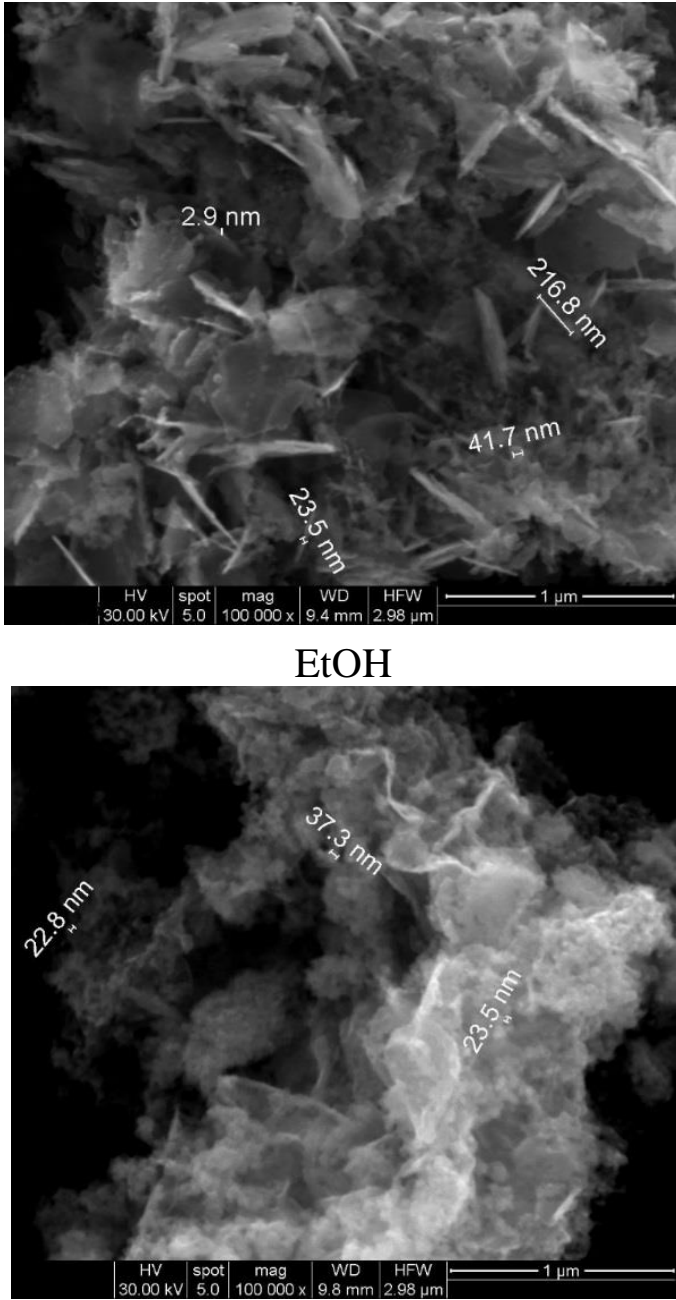

Gly

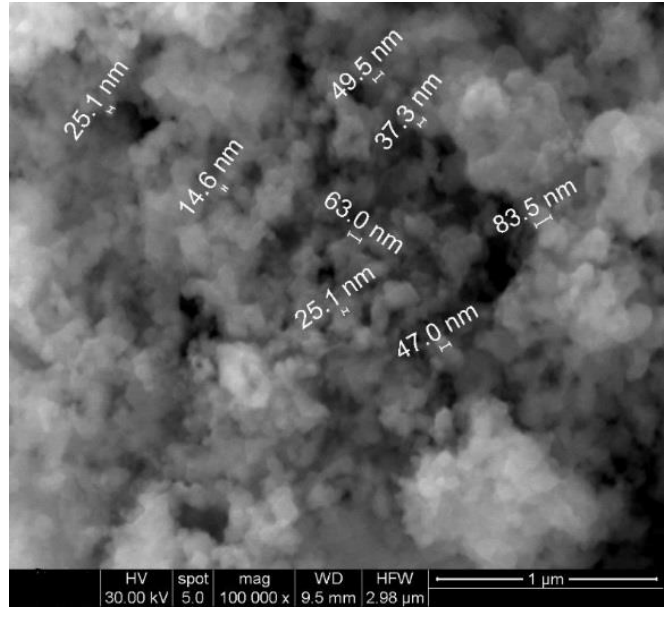

Et O Ac

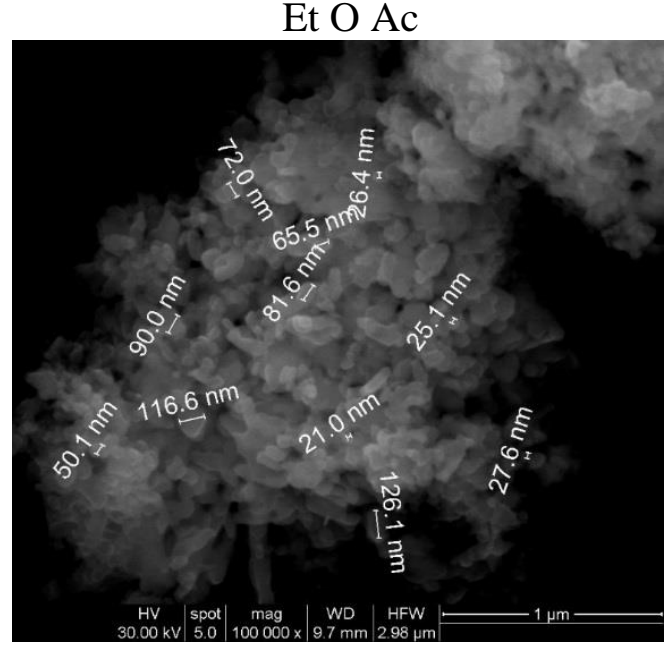

Tol

Figure 4. SEM images of the samples

The images highlight the very important influence of the solvent used to achieve the organizing of the final product. The characteristic morphology of LDHs, well-known in the literature, consists of associations of thin disks with more or less ordered perimeter after all directions, giving association with tagetes flower-like morphology [18]. In the case of the samples from this study, obtaining the samples in non-aqueous solvents generates this feature only in part for the EtOH sample. The measured thickness of the sheets was $2.9 \mathrm{~nm}$, while the planar sheet size varies widely between tens to hundreds of $\mathrm{nm}$.

The appearance of the Gly sample is somehow similar, the sheets united to each other are present in high number, but there is a strong trend to form rather chains of disks covered with many pseudospherical grains of 25-35 $\mathrm{nm}$ in diameter.

The treatment on ethyl acetate (EtOAc sample) results in the formation of a mixture of particles very irregularly shaped and having different sizes, from 10 up to $100 \mathrm{~nm}$.

The Tol sample is made of particles with irregular shapes and quite different sizes. The particles are less agglomerated however than in the case of EtOAc sample.

It is worthy to mention that the use of hydrophilic/water soluble solvents (ethanol and glycerol) generated particles having in a certain extent the same morphology as the product synthesized in water medium, while the use of hydrophobic solvents drastically changed the usual morphology. 


\section{Conclusions}

The synthesis of $\mathrm{ZnFeAl} \mathrm{LDH}$, with $\mathrm{Zn} / \mathrm{Fe} / \mathrm{Al}$ molar ratios of $2 / 0.5 / 0.5$ was synthesized by the coprecipitation method, at a constant $p \mathrm{H}$ value of 8.5. The use of several organic solvents (ethanol ethyl acetate, glycerol and toluene) during the coprecipitation procedure produced important changes of their structural and micromorphological properties.

The XRD patterns confirm the formation of the layered structure for all the samples. The samples prepared with toluene and glycerol were less crystalline than those prepared with ethanol and ethyl acetate while the interlayer space is expanded in the case of the samples prepared with glycerol and ethyl acetate. The particles sizes and their interconnection pattern are also a function of the nature of the solvent.

The FT-IR spectra highlighted the main species from the interlayer. The nitrate anions from the interlayer of the samples prepared from nitrates have been almost totally replaced by carbonate, while in the case of the sample prepared using sulfate, in the interlayer some sulfate ions are still present. The band gaps values are in the range of $2.65-3 \mathrm{eV}$ and point out that these solids could be used as photocatalysts, since the activation energy values allows using the natural solar light for the promotion of the electron from the valence band to the conduction band.

The present findings open novel perspectives to efficiently synthesize LDHs with a controlled morphology for applications in environmental cleanup.

\section{References}

1. BADMUS, K. O., TIJANI, J. O., MASSIMA, E., PETRIK, L., Treatment of persistent organic pollutants in wastewater using hydrodynamic cavitation in synergy with advanced oxidation process. Envir. Sci. Poll. Res., 25(8), 2018, 7299-7314.

2. RUSU, L., SUCEVEANU, M., ŞUTEU, D., FAVIER, L., HARJA, M., Assessment of groundwater and surface water contamination by landfill leacheate: a case study in Neamt county, Romania, Envir. Eng. Manag. J., 16(3), 2017, 633-641.

3. MARGOT, J., ROSSI, L., BARRY, D.A., HOLLIGER, C., A review of the fate of micropollutants in wastewater treatment plants, Wiley Periodicals, Inc, , 2015, 457-487,

https://doi.org/10.1002/wat2.1090.

4. PELIGRO, F. R., PAVLOVIC, I., ROJAS, R., \& BARRIGA, C. (2016). Removal of heavy metals from simulated wastewater by in situ formation of layered double hydroxides, Chem. Eng. J., 306, 1035-1040, https://doi.org/10.1016/j.cej.2016.08.054.

5. BESSAHA, H., HARRATS, C., BOURAADA, M., Demenorval, L.C., Photocatalytic efficiency of ZnAl-layered double hydroxide on degradation of a cationic dye under UV and solar radiation, Chem. Pap. 72, 2018, 1031-1040, https://doi.org/10.1007/s11696-017-0347-9.

6. KHENNICHE, L., FAVIER, L., BOUZAZA, A., FOURCADE, F., AISSANI, F., AMRANE, A, Photocatalytic degradation of bezacryl yellow in batch reactors-feasibility of the combination of photocatalysis and a biological treatment, Envir. Technol., 36(1), 2015, 1-10,

https://doi.org/10.1080/09593330.2014.934740.

7. XIE, Y, YUAN, X., WU, Z., ZENG, G., JIANG, L., PENG, X., LI, H., Adsorption behavior and mechanism of $\mathrm{Mg} / \mathrm{Fe}$ layered double hydroxide with $\mathrm{Fe}_{3} \mathrm{O}_{4}$-carbon spheres on the removal of $\mathrm{Pb}$ (II) and $\mathrm{Cu}(\mathrm{II})$, J. Coll. Interf. Sci., 536, 2019, 440-455.

8. CARJA, G., NAKAMURA, R., NIIYAMA, H., Tailoring the porous properties of iron containing mixed oxides for As (V) removal from aqueous solutions, Microp. Mesop. Mater., 83 (1-3), 2005, 94100, https://doi.org/10.1016/j.micromeso.2005.03.014.

9. CARJA, G., DARTU, L., OKADA, K., FORTUNATO, E., Nanoparticles of copper oxide on layered double hydroxides and the derived solid solutions as wide spectrum active nanophotocatalysts, 2013, Chem. Eng. J., 222, 2013, 60-66, https://doi.org/10.1016/j.cej.2013.02.039. 
10. COZMA, D.G., COSTULEANU, C.L., SANDU, I., LUTIC, D., Statistical analysis of raw and potable water quality from a preparation plant, as a basis to purpose the re-schedule of the physicochemical current routine analysis periodicity, Rev. Chim., 69(1), 2018, 214-221.

11. XU, H., WANG, W., ZHU, W., Shape evolution and size-controllable synthesis of $\mathrm{Cu}_{2} \mathrm{O}$ octahedra and their morphology-dependent photocatalytic properties, J. Phys. Chem. B, 2006, 110(28), 1382913834, https://doi.org/10.1021/jp061934y.

12.THIRUMALAIRAJAN, S., GIRIJA, K., HEBALKAR, N. Y., MANGALARAJ, D., VISWANATHAN, C., \& PONPANDIAN, N., Shape evolution of perovskite $\mathrm{LaFeO}_{3}$ nanostructures: a systematic investigation of growth mechanism, properties and morphology dependent photocatalytic activities, $R S C A d v$., 3(20), 2013, 7549-7561.

13. GILEA, D., RADU, T., MURESANU, M., CARJA, G., Plasmonic photocatalysts based on silver nanoparticles - layered double hydroxides for efficient removal of toxic compounds using solar light, Appl. Surf. Sci., 444, 2018, 407-413, https://doi.org/10.1016/j.apsusc.2018.03.054.

14. LUTIC D., COROMELCI-PASTRAVANU C.G., CRETESCU I., POUliOS, I., STAN C.D., Photocatalytic treatment of Rhodamine 6G in wastewater using photoactive $\mathrm{ZnO}$, Int. J. Photoenergy, 2012, ID 475131, doi:10.1155/2012/475131.

15. SEFTEL, E. M., COOL, P., LUTIC, D., Mg-Al and $\mathrm{Zn}-\mathrm{Fe}$ layered double hydroxides used for organic species storage and controlled release, Mater. Sci. Eng. C, 33(8), 2013, 5071-5078.

16. BARRIGA, C., JONES, W., MALET, P., RIVES, V., ULIBARRI, M.A., Synthesis and Characterization of Polyoxovanadate-Pillared Zn-Al Layered Double Hydroxides: An X-ray Absorption and Diffraction Study Inorg. Chem., 37, 1998, 1812-1820.

17. SURAM, S. K., NEWHOUSE, P. F., GREGOIRE, J. M., High throughput light absorber discovery, part 1: an algorithm for automated Tauc analysis. ACS Combinatorial Science, 18(11), 2016, 673-681.

18. CARJA, G., NAKAMURA, R., AIDA, T., NIIYAMA, H., Textural properties of layered double h, ydroxides: effect of magnesium substitution by copper or iron, Microp. Mesop. Mater., 47(2-3), 2001, 275-284.

Manuscript received: 24.05 .2020 